\title{
Multilinguales
}

\section{Éros, Thanatos et engagement politique de la femme dans Oran, langue morte d'Assia Djebar}

Eros, Thanatos and Women's Political Commitment in Assia Djebar's Oran, Langue Morte

Denise Brahimi

\section{(2) OpenEdition}

\section{Journals}

Édition électronique

URL : http://journals.openedition.org/multilinguales/811

DOI : 10.4000/multilinguales.811

ISSN : 2335-1853

Éditeur

Université Abderrahmane Mira - Bejaia

Référence électronique

Denise Brahimi, «Éros, Thanatos et engagement politique de la femme dans Oran, langue morte d'Assia Djebar », Multilinguales [En ligne], 6 | 2015, mis en ligne le 01 décembre 2015, consulté le 17 septembre 2019. URL : http://journals.openedition.org/multilinguales/811 ; DOI : 10.4000/ multilinguales.811

Ce document a été généré automatiquement le 17 septembre 2019.

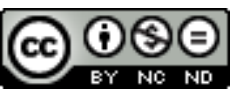

Multilinguales est mise à disposition selon les termes de la Licence Creative Commons Attribution Pas d'Utilisation Commerciale - Pas de Modification 4.0 International 


\section{Éros, Thanatos et engagement politique de la femme dans Oran, langue morte d'Assia Djebar}

Eros, Thanatos and Women's Political Commitment in Assia Djebar's Oran, Langue Morte

Denise Brahimi

1 Dans l'œuvre d'Assia Djebar, l'année 1997 a ceci de remarquable qu'elle correspond à la publication de deux livres, l'un et l'autre aux éditions Actes Sud, d'une part Les Nuits de Strasbourg et d'autre part Oran, langue morte.

On a d'abord l'impression qu'une coupure importante passe entre les deux (quel que soit leur ordre de publication) comme si, avec le second on entrait dans la série de livres dramatiquement inspirés à l'auteure par les événements de la terrible décennie 90 en Algérie. Il est certain en effet qu'ils sont extrêmement présents dans les deux ensembles de nouvelles qui y figurent, dont on pourrait prendre pour exemple en raison de son titre l'une de celles qui se trouvent dans la première partie et qui s'intitule « L'Attentat».

Mais à la réflexion, on se dit que l'écart creusé par cette intrusion de la mort au quotidien ne change sans doute pas profondément la ou les problématiques omniprésente(s) à travers l'œuvre et c'est ce qu'on aimerait faire apparaître ici à partir d'une nouvelle en particulier, celle qui se trouve juste avant «L'attentat » et qui a pour titre « La fièvre dans des yeux d'enfant ».

Une manière de la présenter d'abord très sommairement consiste à dire qu'il s'agit encore et toujours pour l'auteure d'entrelacer d'une part son analyse du désir amoureux et, d'autre part, celle de l'engagement -non moins important à ses yeux voire primordial - dans une action collective qu'il est un peu restrictif de désigner comme politique.

2 Si l'on veut préciser d'emblée les deux termes de cette équation, on peut, d'une part, s'agissant du premier, substituer désir érotique à « désir amoureux », pour insister sur la place de la sexualité, et pour que le mot Eros contenu dans érotique puisse être associé 
plus facilement au mot Thanatos (la mort en grec), tant il est vrai que le célèbre et mythique couple d'Eros et Thanatos est extrêmement présent dans l'œuvre d'Assia Djebar; et d'autre part, s'agissant du second, la notion d'engagement, dont la formulation paraît un peu datée puisqu'il faut la faire remonter à Jean-Paul Sartre (à propos de la seconde guerre mondiale), on peut le compléter en parlant d'« engagement existentiel », puisqu'il s'agit d'un choix de vie allant bien au-delà du militantisme ordinaire, jusqu'à une prise de risque totale, incluant le risque de mort, et ce, en toute conscience, sans que soit pourtant exclue la présence d'une pulsion suicidaire (qui elle, comme toute pulsion, relève de l'inconscient).

\section{Eros / Thanatos et ...}

3 C'est donc à partir de la nouvelle qu'on va essayer de voir ce qu'il en est de l'amour et de l'engagement, au risque de détruire la riche complexité de ce rapport qui fait le tissu littéraire de «La fièvre dans des yeux d'enfant ».

Ce texte, qui fait environ soixante-dix (70) pages, peut être considéré comme une longue nouvelle, et comme c'est généralement le cas chez Assia Djebar, elle ne se réduit pas à un seul moment ni à un seul acte.

Le déroulement du récit n'est pas chronologique : il comporte une circulation narrative entre l'avant et l'arrière, et comme il va au-delà de la mort de l'héroïne principale prénommée Isma, qui en est longtemps la narratrice (sous la forme d'un journal adressé à une amie morte et d'une lettre destinée à un amant), on peut considérer que les deux personnages masculins qui lui survivent, principalement son mari Ali, sont loin d'être secondaires.

Nous savons par maints exemples que cette romancière (mais dans ses nouvelles aussi bien) s'intéresse avant tout aux réfractions d'une situation donnée sur tout un groupe et que leur ensemble se substitue souvent à ce que chez d'autres auteurs on appelle l'action.

Il est donc tout à fait légitime de chercher à comprendre ce qu'il en est de l'amour pour Ali, le mari d'Isma, incontestablement amoureux de sa femme, y compris au sens que nous avons appelé érotique du mot. Et nous sommes aidés dans cette recherche par le fait qu'Isma analyse avec beaucoup de finesse et de précision ce qu'il en est du comportement érotique de son mari.

Elle le fait à partir des événements effroyables qui ont marqué la vie de cet homme alors qu'il n'était encore qu'un enfant : on a ramené dans la maison familiale, et il a eu sous les yeux, le couple de ses père et mère assassinés et sans doute torturés pendant la guerre d'Algérie :

Je ne retournai pas au village ces deux dernières années, à quoi bon? Quand nous montions, je semblais déranger leur tête-à-tête, entre la vieille Selma et Ali. La distance entre lui et moi, la fissure que je décelais désormais, peut-être venait-elle de plus loin ? De la mort de sa mère et de son père. Il n'en parlait jamais. J'avais su, presque par hasard, l'horreur de cette mort: les deux corps ramenés à l'aube chez Selma, deux corps fusillés et, disait-on, avec des traces visibles de torture, sur l'un et sur l'autre, avec en outre (mais cela, une parente lointaine de passage me l'avait hasardé une fois, là-bas) les preuves d'un viol qu'avait dû subir auparavant la jeune morte. Or l'enfant, oui, leur garçon (Ali à six ans) avait contemplé en silence les deux dépouilles. (p. 98) 
De ce fait, il a été élevé par sa grand-mère, une vieille dame kabyle qui, au moment où commence la nouvelle, si on la remet dans l'ordre chronologique (trois ans avant son dénouement), est en train de mourir en présence de son petit-fils Ali, mais en l'absence de sa belle-fille Isma.

Ce qui nous est dit alors à propos de cette mort est double. D'une part, tout se passe comme si Ali voulait être seul pour vivre cette nouvelle mort qui vient marquer sa vie ; il remet à plus tard le moment où socialement Isma sera présente à ses côtés pour partager un peu l'événement mais de façon superficielle : "A l'aube, alors que je faisais ma valise (...), Ali m'appela: - Non ne monte pas! C'est de toute façon trop tard. Nous l'avons inhumée hier !...(..) Tu viendras au quarantième jour, si tu y tiens! ajouta-t-il brièvement avant de raccrocher. (p. 104)

Mais d'autre part, il a frénétiquement besoin d'elle au moment de cette mort, et besoin de partager avec elle tout un érotisme qui est comme une réponse ou un démenti à la mort :

Comme si d'en haut, surplombant de très loin nos deux corps, je cernais son désir sans vrai désir, son désir-appel-au-secours, cet appel muet de l'enfant de l'enfant de six ans, tandis que son corps musclé et noueux d'homme de quarante ans reniait, pour la première fois, la morte de Béni-Ourtilane. (p. 108)

C'est sans doute là l'une des raisons pour lesquelles il faut qu'Isma s'en tienne à l'écart et incarne l'autre versant d'un monde où Ali pourra survivre, en opposant Eros à Thanatos.

C'est peu de dire qu'Ali aime Isma, mais il semble pourtant que cette dernière ait du mal à accepter l'idée que l'amour pour elle est un moyen, le seul qu'Ali ait pu trouver dans son combat contre les assauts renouvelés de la mort qui s'acharne au plus près de lui. Et qui s'acharnera finalement une dernière fois en lui prenant Isma.

La complexité des sentiments est telle, dans les œuvres de Djebar, qu'on peut aller très loin dans les suppositions. Isma nous dit ce qu'elle croit comprendre, c'est-à-dire que cet amour que lui porte Ali ne la concerne pas vraiment : même si elle se sait aimée, ce n'est pas vraiment d'elle qu'il s'agit.

Il y a là une indication bien intéressante sur l'exigence que représente une telle conception de l'amour, que ce soit celle de l'auteure elle-même ou celle de son personnage : l'amour passe par la connaissance et la reconnaissance de la personne aimée, la question qui, inévitablement, est en son cœur est : qui es-tu ? Qui suis-je ? Car on ne se trouve pas dans l'autre, mais c'est l'autre qui nous aide à nous trouver; la conséquence étant que, s'il ne peut jouer ce rôle, il ne peut sans doute pas y avoir d'amour, au sens plein, voire absolu, du terme :

Il pénétra dans notre chambre, l'après-midi. Il me prit par la main. Il me dirigea vers le lit (...) Que suis-je, en cet instant, dans ses mains d'aveugle? Une geisha? Non, c'est de moi qu'il a besoin, je le sens, (...). Il m'appelle, il vient à moi sans mots et ses caresses quémandent, mais quoi... (p. 106)

Naturellement toute affirmation de cette sorte paraît très ou trop catégorique, mais on constate du moins à quel point l'amour est difficile et fragile; et comme à cela il faut trouver des explications, le texte littéraire les suggère mieux que tout autre parce qu'il rationalise moins que le discours analytique.

Isma dit qu'elle aime Ali comme une amie et comme une sœur (p.105), et en même temps elle n'a qu'une idée en tête, se séparer de lui pour vivre seule, ce qu'elle parvient bientôt à faire, pour d'autres raisons encore dont on reparlera à propos de son engagement. 
La raison qui concerne l'amour est que justement, l'amour au sens le plus exigeant du terme, pour Ali elle n'en a sans doute pas, elle n'en ressent pas sous la forme positive qui lui ferait désirer vivre avec lui.

L'obstacle est certainement la vie en couple qui concrètement amène une femme comme Isma à subir l'amour physique sans qu'elle ait l'impression de le vouloir et de le choisir :

Je le suis. Je ne dis rien. Je suis durcie à l'intérieur, mais je reste attentive à ses gestes retenus, contraints. (... Allongée, en robe légère, je le regarde : "il revient vraiment des franges de la mort", me dis-je toujours durcie, sans penser à moi. Je l'accueillerai : il paraît tout silence. (p. 107)

Cette inégalité profonde de la situation traditionnelle a des effets néfastes et en particulier parce que pour nombre d'héroïnes ou narratrices de Djebar qui sont ses porte-paroles, le désir se trouve rejeté dans la sphère extra-conjugale, où il est voué à un statut éphémère et pour ainsi dire irréalisable.

Mais pour parler encore du rapport entre Isma et Ali au moment où commence chronologiquement cette histoire, et pour en revenir à l'idée que face à tant de complexité, toutes les suppositions sont permises, il n'est pas impossible qu'il y ait une part de prétexte dans les motivations et analyses données par Isma. Il y a ce qu'elle dit et que nous venons d'évoquer: Isma ne l'aime pas pour elle-même, en pleine connaissance d'elle-même, et à sa manière - bien qu'il soit loin d'incarner l'homme dominateur traditionnel trouvant sa raison d'être dans l'affirmation du droit conjugal - il n'en exploite pas moins la situation de couple.

Elle reconnaît que dans l'état de détresse existentielle où il se trouve, c'est le seul moyen de survivre à sa disposition mais pour autant son amour à elle ne peut résister à son « utilisation».

Cependant et même si l'on reconnaît que ce dernier mot (« utilisation ») est l'obsession négative du féminisme, on peut aussi penser -et d'ailleurs Isma ne s'en cache pas-que le déplacement de son désir vers un ailleurs ou vers un autre a pris sens et effet à partir de sa rencontre avec l'étranger musicien, le Somalien Omar qui va incarner pour un temps du moins son désir érotique au féminin. Beaucoup de circonstances contribuent à cette cristallisation comme dirait Stendhal ${ }^{1}$ : la musique, la poésie et une certaine qualité de la langue arabe (notamment sa sonorité, si importante pour les héroïnes de Djebar) :

Naquit une camaraderie entre nous, prompte, une affinité que je sentais innocente, sans nulle ambiguïté... s'il n'y avait eu ce tangage de ses accents, ce frôlement, par moment, et par bribes de la langue arabe...j'écoutais intensément, quelque chose en moi s'arrachait. (pp. 86-87)

Dans le jeu de familiarité-étrangeté si propice à la naissance du désir, Omar représente sans doute pour Isma un composé idéal. Bien que nous sachions peu de chose de leur relation, il semble qu'il ait gardé jusqu'au bout pour elle un certain caractère énigmatique et non élucidé.

Reste que le désir d'Isma pour Omar, si explicable qu'il soit et favorisé par le fait qu'à ce moment Ali est tout entier englouti dans le deuil et l'amour des morts (de la mort), ce désir donc, comme tout autre, garde une part d'imprévisible lié à son surgissement aléatoire et à son absence d'insertion dans le temps.

Le rationnel (ou rationalisable) et l'irrationnel coexistent sans doute au sein du désir, de manière à en faire un objet d'étude particulièrement fascinant, comme on le voit par exemple dans l'importance qu'il a prise pour le psychanalyste Lacan. 
Aux confins de ces deux aspects, il y a peut-être (car en littérature on ne peut être sûr de rien) cet amour de jeunesse, ou premier amour, que lui avait voué un certain Omar, du même prénom que le musicien et dont on ne sait au juste à quel degré d'oubli elle le portait en elle jusqu'au moment où il ressurgit, opportunément, pour justifier qu'elle emploie ce prénom en présence de son mari. Peut-être ressurgit-il parce que quelque chose de très ancien s'est trouvé réactivé par la rencontre avec le nouvel Omar :

Je sais qu'Ali, à peine émergé de son plaisir (...), je sais simplement qu'il entendit une voix, ma voix - or celle-ci me trahit - elle laissa échapper assez haut, distinctement un mot (...) - Omar ! Ali se figea (...). Soudain, l'illusion se présente, secourable, (...) pour calfeutrer ce trou ouvert par un seul mot. Oui, bien heureusement, je me rappelle (...), Ali, je le sens, s'est rappelé aussi (...) : l'anecdote dont nous avions tant de fois, ri, aux premiers temps de nos amours. J'avais décrit "mon premier amoureux du lycée" (...) : Omar, il s'appelait Omar. (p. 109)

Ruse ou pas de la part d'Isma, on est amené à constater qu'Omar se dédouble et trouve son insertion dans le passé en même temps qu'il est l'homme neuf dans une situation où cette nouveauté est à la fois inattendue et profondément désirable.

Quelle forme prend le désir d'Isma pour Omar? Alors que de la part d'Ali, on a l'impression qu'il s'agit d'une séduction à la manière poético-orientale.

Sans qu'on sache jamais l'importance ni la force de son sentiment (ni même si sentiment il y a ), on est beaucoup mieux informé sur le fait qu'elle est portée vers lui par une pulsion en partie physique, même si elle ne l'est pas seulement et s'il n'y a pas de violence contraignante dans ce qu'elle ressent.

L'idée de contrainte ne vient pas à l'esprit parce qu'on a, au contraire, le sentiment que cette relation est, de sa part à elle, un choix libre et que c'est l'exercice de cette liberté qui lui donne envie d'avancer dans le sens de sa pulsion. Complexité décidément, amateurs de simplicité s'abstenir! Le Somalien Omar part en Hollande et demande à Isma de l'y rejoindre pour huit jours afin de mener à bien leur travail d'ethnologie musicale. A partir de ce moment, la nouvelle (ou la nouvelliste) profite de la possibilité d'ellipse qui est une caractéristique de cette forme littéraire. On a toute raison d'imaginer que pendant ce séjour, Omar et Isma deviennent amants mais on n'en a non plus aucune preuve, et pas davantage du fait qu'éventuellement ils seraient restés en relation après le retour d'Isma en Algérie.

Ce qui est certain, c'est qu'à ce moment-là Isma se sépare d'Ali comme elle en avait le projet et qu'elle entre dans la clandestinité, se cachant avec le plus grand soin pour échapper aux assassins qui sévissent alors dans le pays et qui massacrent peu après Nawal, la meilleure amie d'Isma et son mentor dans le monde du combat clandestin.

Certain aussi et tout à fait essentiel pour que nous comprenions l'évolution d'Isma, le fait que dans l'avion qui la conduisait vers Omar, elle a écrit pour celui-ci une lettre qu'elle ne lui a pas remise; en fait c'est Ali qui, trouvant cette lettre dans les papiers d'Isma après sa mort, prend l'initiative de l'envoyer à Omar puisque c'est à lui qu'elle était destinée.

Le contenu de cette lettre n'est pas rendu clair par l'auteure, qui laisse à ses lecteurs le soin de beaucoup deviner. Cependant on en sait assez pour comprendre qu'à ce moment-là Isma sait déjà que sa rencontre avec Omar restera sans lendemain.

A cet égard, elle se sent dit-elle en avance sur lui, mais c'est sans doute pour ne pas gâcher leurs " huit jours ", et surtout pas pour Omar, puisqu'elle ne lui remet pas la lettre.

Ces huit jours et ces huit nuits dont on ne saura rien font évidemment penser aux neuf nuits de Strasbourg, dans le roman qui porte ce titre et qui date à peu près du même 
moment, en tout cas de la même année. En tant que romancière exigeante, elle ne va pas reprendre deux fois le même livre, pas même pour intégrer partiellement le roman dans la nouvelle.

En fait ce qui compte dans la nouvelle d'Oran, langue morte est ce qui va se passer après le retour d'Isma en Algérie et à partir de son entrée dans la clandestinité.

\section{... Engagement politique}

6 Ici commence notre volonté de comprendre ce qu'il en est pour Assia Djebar de l'engagement ou engagement existentiel, ce qui en un sens nous ramène bien en deçà dans le déroulement de son œuvre, retournant vers ce livre qui a fait le début de sa célébrité, c'est-à-dire Les Alouettes naïves de 1967.

Sur l'action clandestine, et c'est d'ailleurs vrai dans les deux cas, on n'apprend à peu près rien, sinon que, dans la nouvelle publiée en 1997, il s'agit de défendre la cause des femmes face à l'islamisme radical et meurtrier :

J'allais à la seconde manifestation des femmes (...). Cette fois pas seulement les femmes, mais des intellectuels, des étudiants, des familles avec les enfants, (...) défilaient dans un désordre presque bon enfant: (...) pour la sauvegarde des femmes qui travaillaient, elles que des loubards, prétendument au nom de la religion, agressaient dans des petites villes du Sud, du Nord. (p. 104)

Dans la construction narrative de la nouvelle, c'est par la description de cette Isma solitaire et soigneusement déguisée en « vieille tricoteuse » anonyme que commence le récit. On saura plus tard qu'elle ne donne alors aucune nouvelle d'elle, pas même ou surtout pas à Ali qu'elle veut protéger (encore une des raisons pour lesquelles elle s'est séparée de lui). Et naturellement elle se garde bien aussi d'aller à l'enterrement de son amie Nawal qui a été assassinée avant qu'elle ne le soit elle-même.

D'une certaine manière et pour ce qui concerne la relation d'Isma avec Ali, la solitude de son engagement est alors une sorte de réponse à la solitude dans laquelle Ali a voulu vivre la mort de sa grand-mère le ramenant en arrière à celle de ses parents.

Il en ressort, ou il pourrait en ressortir, que l'engagement n'est pas compatible avec l'amour mais on dira peut-être que, de l'amour, chez Isma, il n'y en avait pas vraiment. Cependant l'engagement n'est pas davantage compatible avec le désir érotique qu'elle éprouvait pour Omar. C'est en cela que la position de la femme a changé pendant les trente ans (1967-1997) qui se sont écoulés depuis Les Alouettes naïves.

Dans ce roman-là, c'était l'homme Rachid qui ne parvenait pas à assurer la coexistence des deux, tandis que la femme, Nfissa voulait absolument la réaliser.

Il semblerait que la romancière en soit arrivée, après tant d'années, à la conviction que l'engagement ne peut être vécu ni dans l'amour au sein du couple, ni dans la relation érotique, d'où le sentiment du tragique qui se développe dans son œuvre - puisque tragique il y a chaque fois que s'affirme l'incompatibilité entre deux aspirations aussi essentielles l'une que l'autre, au point qu'on ne peut renoncer à aucune d'entre elles deux sans mutilation, c'est-à-dire, à terme, sans risque de mort.

Lorsqu'elle revient de Rotterdam, Isma n'ignore évidemment pas la manière quasi inévitable dont se terminera l'engagement qu'elle est en train de prendre. Faut-il alors parler d'une pulsion suicidaire qui viendrait alors s'ajouter à son incontestable désir de servir la cause urgente et indiscutable en laquelle elle croit?

On sait, notamment depuis la parution de son dernier roman Nulle part dans la maison de mon père (2007), à quel point cette pulsion a été importante dans la vie d'Assia Djebar, 
présente et agissante dès son entrée dans la vie, mais certainement de manière inconsciente, puisqu'elle n'arrive pas à s'en expliquer elle-même encore bien longtemps après.

7 Le rapprochement avec ce dernier roman, évidemment postérieur à Oran, langue morte (puisqu'il est de 2007), met sur la voie d'une explication qui n'est pas dite dans la nouvelle mais qui pourrait y être implicitement. Le point commun aux deux situations évoquées serait un sentiment indicible ou en tout cas non dit de culpabilité, qui pour s'en tenir à la nouvelle paraît d'abord relativement facile à expliquer et à localiser :

En ce temps là, chaque jour m'apportait sa nouvelle luisante de suie (...), sa nouvelle de mort (...)

Pourquoi l'évoquer et pourquoi prendre la plume : désespoir de survivre, dégoût de parler ou de dire ?...Mais d'écrire ? En catimini, en secret, dans un coin, comme un marmonnement de fou ou de solitaire ? Quand tout de ces lieux sera détruit et que ne resteront, de moi en tout cas, que ces griffonnements sur un cahier jeté, oublié, dont l'auteur à son tour sera... (pp. 71-72)

8 Etant donné qu'au moment où elle prend cet engagement, elle vient de vivre une aventure érotique extraconjugale, en partant pour cela rejoindre au loin un étranger, étant donné d'autre part, qu'au retour de cette péripétie audacieuse elle a quitté son mari pour aller vivre ailleurs et apparemment sans lui donner l'explication de cette décision non moins audacieuse, on est en droit de se dire, même si l'on n'est pas spécialement moraliste soi-même, qu'une sorte de surmoi moral a fait en elle une apparition forcément culpabilisante.

Sur le même modèle et pour développer au moins un peu la comparaison avec Nulle part dans la maison de mon père, on voit bien dans ce dernier roman la culpabilité que la narratrice ressent et principalement à l'égard de son père qui l'a toujours poussée à s'émanciper intellectuellement, du fait qu'elle profite de la liberté de mouvement qu'il lui accorde pour entretenir une liaison amoureuse clandestine. Elle n'a de cesse de répéter vers la fin du roman: «Si mon père l'apprend, je me tue!»(p. 430); "Si je suis convoquée au tribunal du père...je me tue!» (p. 431); «Si mon père ... je me tue!» (idem) ; «si mon père le sait, je me tue » (p. 433); «Si mon père le sait...je me tue!» (p. 435).

Mais la culpabilité à l'égard d'un mari ou d'un père est-elle suffisante pour expliquer une pulsion suicidaire, désir de mort qui dans le cas de la nouvelle est malheureusement suivi d'effet, tant il est vrai que les circonstances s'y prêtent.

A y regarder de plus près, on est amené à chercher plus loin et au-delà les raisons d'une culpabilité plus secrète et plus profonde.

Pour s'en tenir cette fois à la nouvelle d'Oran, langue morte, ce dont Isma se sent coupable vis à vis d'Ali n'est peut-être pas de l'avoir trompé avec un autre homme, comme on dit très banalement, mais plutôt de ne pas avoir été capable de mener avec lui un double projet (et de ne pas même l'avoir tenté), celui de l'amour conjugal et celui de l'engagement existentiel dans un projet :

Nous gagnerons! déclara Ali, ce même soir, rentrant de l'hôpital, tandis que je lui rapportais la scène de l'adolescent au crachat, mais aussi le défilé impressionnant de nos sympathisantes. Sur quoi, il partit au village de montagne. (pp. 102-103)

Oran, langue morte marquerait ainsi une dissociation patente au sein de la grande formation mythique qui a soutenu la démarche d'Assia Djebar pendant des décennies depuis l'époque des premiers romans.

Il s'agit pour elle d'une sorte de mythe qu'on peut dire originel, puisqu'il se trouve dès le début de son œuvre, qui est d'associer dans une même volonté et pratique de l'émancipation féminine, l'amour et l'engagement. Comme pour tout mythe, il y a peut- 
être ou certainement eu au départ, ce qui a pu lui apparaître comme une réalité, celle qu'elle vivait au moment de son mariage et pendant la guerre d'Algérie.

Mais il semble qu'à partir des années 80 et peut-être même déjà auparavant, la part du réel s'est amenuisée, voire effacée, au profit du fantasme, lui laissant de plus en plus ce sentiment d'échec qui est à l'origine du désir de mort.

La nouvelle prend acte du fait que, décidément et définitivement, l'association rêvée est irréalisable. Concrètement dans le récit, l'échec se traduit par la mort de la narratrice, une mort qu'elle n'a pas eu besoin de se donner elle-même (à la différence de la tentative racontée dans Nulle part dans la maison de mon père) puisque d'autres s'en sont chargés.

Les deux hommes de la nouvelle, Ali et Omar, survivent mais n'ont rien pu pour elle. En choisissant l'engagement, elle a choisi, de manière explicite, de tourner le dos à l'éros : les premières pages sont consacrées au vieillissement et à l'enlaidissement volontaire d'Isma ; et au même moment, ou juste avant, elle avait choisi aussi de mourir à l'amour conjugal, sorte de mort symbolisée par le fait qu'elle quitte le domicile conjugal sans vouloir garder le moindre lien (même de proximité) avec son mari.

Sur l'inévitable dissociation, traduite dans la langue littéraire d'Assia Djebar par les mots de "déchirement" ou d' "écartèlement ", on trouve dans la quatrième de couverture d'Oran, langue morte une formule très remarquable, apparemment appliquée à l'Algérie, pour dire l'intention du livre : "Qu'ai-je cherché (...) dans la seule Algérie, tandis qu'elle est de plus en plus écartelée entre désir et mort?».

On est tenté de reprendre la formule pour l'appliquer à l'auteure elle-même, d'autant qu'elle évoque ensuite sa volonté d'écrivaine qui est de dire le drame vécu par ses sœurs pourchassées et meurtries.

C'est l'auteure elle-même qui se sent écartelée, interdite de désir pour pouvoir mieux se consacrer à la défense de ses sœurs.

\section{BIBLIOGRAPHIE}

DJEBAR A., Les Alouettes naïves, Julliard, 1967 ; Actes Sud « Babel », 1997.

DJEBAR A., Oran, langue morte, Actes Sud, 1997. Djebar A., Les Nuits de Strasbourg, Actes Sud, 1997.

DJEBAR A., Nulle part dans la maison de mon père, Fayard, 2007.

SARTRE J.-P., Qu'est-ce que la littérature ?, Gallimard, 1948.

STENDHAL, De l'amour, I, chap. II, Librairie universelle, 1822. Disponible sur le site : < http:// gallica.bnf.fr/ark:/12148/bpt6k68866 > 


\section{NOTES}

1. «Ce que j'appelle cristallisation, c'est l'opération de l'esprit, qui tire de tout ce qui se présente la découverte que l'objet aimé a de nouvelles perfections", dans Stendhal,De l'amour, I, chap. II, Librairie universelle, 1822, p. 8.

\section{RÉSUMÉS}

Dans cette réflexion autour de la nouvelle «La Fièvre dans des yeux d'enfant » du recueil Oran, langue morte d'Assia Djebar, nous montrons qu'il s'agit encore et toujours pour l'auteure d'entrelacer désir amoureux et engagement politique. Nous avons donc cherché à comprendre ce qu'il en est de l'amour et du désir d'Isma pour son mari Ali et son amant Omar, confrontés à son engagement pour la cause des femmes menacées par les intégristes. Nous aboutissons à la conclusion que pour la romancière l'engagement n'est compatible ni avec l'amour au sein du couple, ni avec la relation érotique ; d'où le destin tragique du personnage féminin ici représenté par Assia Djebar.

In this reflection on the short story: "La fièvre dans les yeux d'enfant" from AssiaDjebar's collection Oran, langue morte, we show that the author is still interweaving loving desire and political commitment. So we tried to understand Isma's love and desire for her husband Ali and her lover Omar, faced with her commitment to the cause of women threatened by the fundamentalists. We have reached the conclusion that to the novelist, commitment is neither consistent with love between the couple nor with the erotic relationship ; hence the tragic fate of the female character here represented by AssiaDjebar .

\section{INDEX}

Mots-clés : engagement, tragique, La Fièvre dans des yeux d'enfant, Oran, langue morte, Assia Djebar

Keywords : commitment, tragic, La fièvre dans les yeux d'enfant, Oran, langue morte, Assia Djebar 